Published as: Widener, M.J., Farber, S., Neutens, T. \& Horner, M.W. (2013). Using Urban Commuting Data to Calculate a Spatiotemporal Accessibility Measure for Food Environment Studies. Health \& Place, vol. 21, p. 1-9.

\title{
Using Urban Commuting Data to Calculate a Spatiotemporal Accessibility Measure for Food Environment Studies
}

\begin{abstract}
Improving spatial access to healthy foods in urban regions is recognized as an important component of reducing the prevalence of chronic illness and achieving better health outcomes. Previously, researchers exploring this domain have calculated accessibility measures derived from the travel cost from home locations to nearby food stores. This approach disregards additional opportunities that present themselves as residents move throughout the city. A time-geographic accessibility measure is utilized to explore how single-occupancy automobile commuting affords access to supermarkets. Results show residents in some TAZs have more access when accounting for their commuting behavior than when measuring access from their home. This finding suggests more nuanced calculations of accessibility are necessary to fully understand which urban populations have greater access to healthy food.
\end{abstract}

Keywords: Food Deserts, Time Geography, Commuting Patterns, Access, Cincinnati 


\section{Introduction}

Spatial accessibility to healthy foods is an important aspect of encouraging nutritious diets as a means to reduce the prevalence of chronic illnesses, such as diabetes and cardiovascular disease (Cummins and Macintyre, 2002; Hung et al., 2004; Joshipura et al., 2001). Neighborhoods lacking access to vendors that provide healthy food options, like fresh fruits and vegetables, have been labeled "food deserts" (Shaw, 2006; Walker et al., 2010). Food deserts are often associated with socioeconomically disadvantaged populations, which exhibit a range of poor health outcomes, such as increased risk of diabetes (Everson et al., 2002) and stroke (Cox et al., 2006). It is assumed that the poor nutritional quality of locally available foods negatively influences the diets of residents, resulting in these undesirable health outcomes (Hendrickson et al., 2006; Lopez, 2007; Schafft et al., 2009).

However, previous food desert studies have not consistently identified a link between access to healthy foods and better health outcomes (An and Sturm, 2012; Wrigley, 2002). One potential reason for these inconsistent findings could be the failure of previous research to consider the impact of the temporal dimension on accessibility (Widener et al., 2011). Generally, the bases of most food accessibility metrics involve measuring the distances between residents' households to the available food stores in a city (Larsen and Gilliland, 2008; Lee and Lim, 2009; Raja et al., 2008). Such measures provide basic information about which urban regions lack spatial access at a point in time, but neglect to account for how a population's movements constrain the level of access over the course of a day. Omitting these dynamics is problematic because it can distort the true level of spatial access different subpopulations experience, and subsequently misinform policy makers' decisions regarding appropriate interventions (Neutens et al., In Press; Widener et al., 2011).

This paper introduces a time-geographic approach to further understand how the urban spatial structure of daily commuting behavior alters the spatiotemporally static image 
of food deserts. We present a study of Cincinnati, Ohio, a city recognized as having a number of "food deserts" (ERS USDA, 2012). The study utilizes a spatiotemporal measure to produce a novel representation of residents' level of access to healthy foods using data on regional commuting patterns, as opposed to previously derived metrics that only consider access from home locations (with exception to Salze et al. (2011)). Given the daily movements of an urban population, this novel measure can provide new information to public health and transportation policy makers seeking to understand the role spatial access to healthy food plays in population health.

\section{Background}

Physical accessibility to goods and services that aid in improving and maintaining health is recognized as an integral component of the overall level of access a person experiences. While other components, like social and economic factors, are also critical to overall accessibility, physical access is arguably the most rudimentary (US Department of Health and Human Services, 2010). If a person cannot physically travel to a location providing something they need, the other two components become less relevant.

In this paper, a healthy food vendor is defined as a supermarket or a large grocery store with a wide variety of foods, including fresh produce, which are generally not available in smaller grocery and convenience stores in the USA (Powell et al., 2007). The process for selecting supermarkets in this analysis is discussed in more depth in section 3.1. For many studies on access to healthy food vendors, distance and transportation costs are used to understand the level of access experienced by people across various study areas (Alwitt and Donley, 1997; Bertrand et al., 2008; Lee and Lim, 2009; Metcalf and Widener, 2012; Widener et al., 2012). For example, Páez et al. utilize travel survey data to infer activity spaces around low-income residents' households, calculated by modeling the average 
distance of all daily trips, to gauge access to retail and fast food vendors throughout Montreal (Páez et al., 2010).

Much of this research relies on the assumption that the distance to a healthy food vendor is measured from the home location. One exception to this is found in research conducted by Salze et al., who introduce a study that reframes how accessibility to food stores can be calculated (Salze et al., 2011). In their paper, the authors propose a measure that considers the potential for spatial interaction given commuting behaviors in the Bas-Rhin administrative region of France. While this work does provide insights into how travel behavior affects access to food vendors, it does not take into account the importance of the available time a resident has to actually utilize a food store that can be more easily accessed thanks to commuting behavior.

This paper explores how automobile commuting patterns affect spatial accessibility to healthy food stores in Cincinnati, Ohio and expands on Salze et al.'s work by quantifying the amount of time a commuter will have to shop at a food store given the amount of free time available to them after accounting for their commuting costs. In addition to providing a spatiotemporal accessibility measure, this research also offers insights into the single occupant car-commuting population, which made up over three quarters of commuters in the U.S. in 2009 (McKenzie and Rapino, 2011). Concentrating on the automobile commuting population is an important step in understanding how movement in an urban region can influence which residents have access (Horner, 2004), as the regularity of the commuting trip may provide routine opportunities to shop at healthy food stores that are not captured by home-based accessibility metrics. To add context to this study, the American Community Survey finds that approximately $71 \%$ of workers 16 years and over in Cincinnati commute to work by driving alone, while approximately $9 \%$ commute using public transportation (US 
Census 2012). While the focus of this research is on automobile commuters, the study can be replicated for commuters utilizing other modes on their journey-to-work, like public transit.

\section{Methods}

A first step in understanding the dynamics of access to healthy foods in urban environments involves the use of tools capable of quantifying the level of availability given an approximation of the typical time-space constraints on the population. The following section describes an interaction metric that can be used to understand how much access to healthy foods a resident has, given her/his daily commute.

\subsection{Market Interaction Potential}

We apply an interaction potential metric that uses interzonal commuting patterns to generate a healthy food accessibility score based on time-space prims (Farber et al., In Press). This accessibility measure incorporates aggregate travel patterns such as commuter flows and activity constraints. For example, a resident could live in neighborhood $i$, where there is a low level of spatial access to healthy food vendors. However, the same resident could have a daily commute to neighborhood $j$, where there are many healthy food vendors, thus providing an opportunity for healthy grocery shopping. This idea is illustrated in Figure 1. The figure shows two potential space-time prims of an individual: one with co-located anchors at the home location and one with dislocated anchors at work and home. The individual can access both a convenience store and a supermarket along the way from work to home, whereas s/he would only be able to access convenience stores from the home location within the same time budget.

Previous healthy food environment measures are confined to investigating locations of residence alone, thus discounting this second opportunity. However there is evidence that 
some households chain trips when shopping for groceries (Cervero, 1996; Horner and O'Kelly, 2007; Popkowski Leszczyc et al., 2004). With this in mind, it is important to note this approach is meant to explore access to healthy food stores for commuters who are also the primary grocery shopper, and who do not necessarily shop during weekends. Despite this, determining to what extent accessibility exists from these non-residence anchor points allows researchers to reconceptualize the impact of the food environment.

It is possible to quantify the amount of time available for grocery shopping at a healthy food vendor to residents living in zone $i$ and commuting to zone $j$ by using a modified version of the social interaction potential score (SIP) presented in (Farber et al., In Press). Instead of considering the potential for two residents living and working in different zones to interact at some set of hypothetical destinations, as is done by Farber et al., the "supermarket" interaction potential $(S M I P)$ score considers the potential time available to commuters for shopping (interacting) at supermarkets given a time budget, their home location, and their work location. In this research, the amount of interaction possible is limited only by the free time a commuter has available after work.

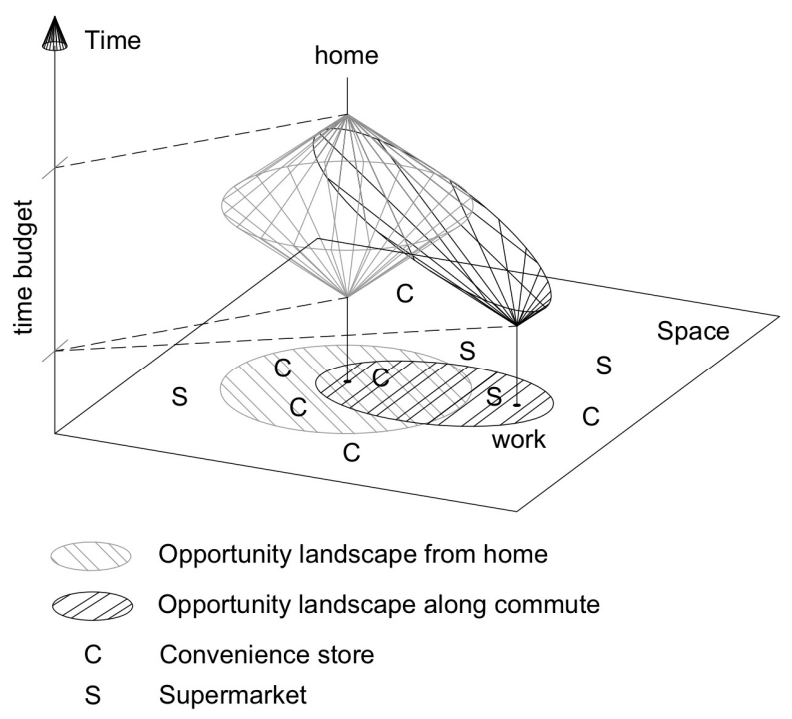

Figure 1. Example of how routine movements may expand a household's access to healthy food. 
The SMIP score is computed for the 359 transportation analysis zones (TAZs) that are within or intersect the city of Cincinnati's boundary. These zones have an average population of $1,237.6$ and a standard deviation of 973.8 . Due to data constraints, in this study we only consider the population of automobile commuting residents. It is important to note the focus of the study is on commuting residents with a home location within the official urbanized area TAZs, but who commute to any of the TAZs in the greater metropolitan planning organization (MPO) region. Commute cost in minutes during peak hours, commuter flow size during peak hours, and demographic data are obtained from the local MPO. The locations of supermarkets throughout the MPO region are obtained through Orbis (2012) and crosschecked through Google Maps directory. The supermarkets used in this analysis are restricted to national and regional supermarket chains like Save-a-Lot, Kroger, Remke Markets, and IGA markets, in addition to the Findlay Market, which serves as a year-round hybrid supermarket/farmers' market in the Over-the-Rhine neighborhood, north of downtown. This restriction is implemented because, while the Orbis dataset is mostly complete, it has two specific issues. First, it includes a few supermarkets that have closed and omits a few new supermarkets that have opened. Second, the Orbis dataset includes convenience stores and gas stations with "supermarket" NAICS codes, despite on the ground confirmation that these stores do not stock a large array of healthy foods, like fresh produce. For more on issues with food environment data see Powell et al. (2007). Ultimately, the collection used in the paper is representative of the supermarkets selling healthy foods available to residents in the Cincinnati metropolitan area, as of the summer of 2012. Supermarket locations are then generalized to their containing TAZ's centroid to maintain consistency with the commuter flow and travel-time data. 
Figure 2 presents a map of the resident TAZs considered for this study, supermarkets locations, and regions designated as food deserts by the US Department of Agriculture (USDA). The USDA defines a food desert as a census tract with a poverty rate at or greater than 20 percent, or a median family income at or less than 80 percent of the area's median family income and have "at least 500 people and/or at least 33 percent of the census tract's population" who reside more than one mile from a supermarket or large grocery store (ERS USDA). While the USDA's method for defining food deserts is straightforward, it is imperfect. One particularly important limitation is their consideration of the urban environment at the tract-level. The aggregation of demographic data and use of coarse distance measures to supermarkets can result in overly generalized results. Despite this drawback, the public and policy makers likely utilize these USDA-designated food desert tracts to determine the locations of food deserts. For this reason, they are included for later comparison.

The SMIP score for a resident living in TAZ $i$ that works in $\mathrm{TAZ}_{j}$ and shops at a supermarket in TAZ $k$, is calculated as:

$S M I P_{i j k}=\max \left(0, B-\left(t_{j k}+t_{k i}\right)\right)$

where $B$ is the free time budget a resident has before they must return home after leaving work, $t_{j k}$ is the travel time in minutes from the work TAZ to the supermarket TAZ, and $t_{k i}$ is the travel time from the supermarket TAZ to the home TAZ. In other words, $S M I P_{i j k}$ represents the number of minutes a person has to shop for groceries after work, given they 


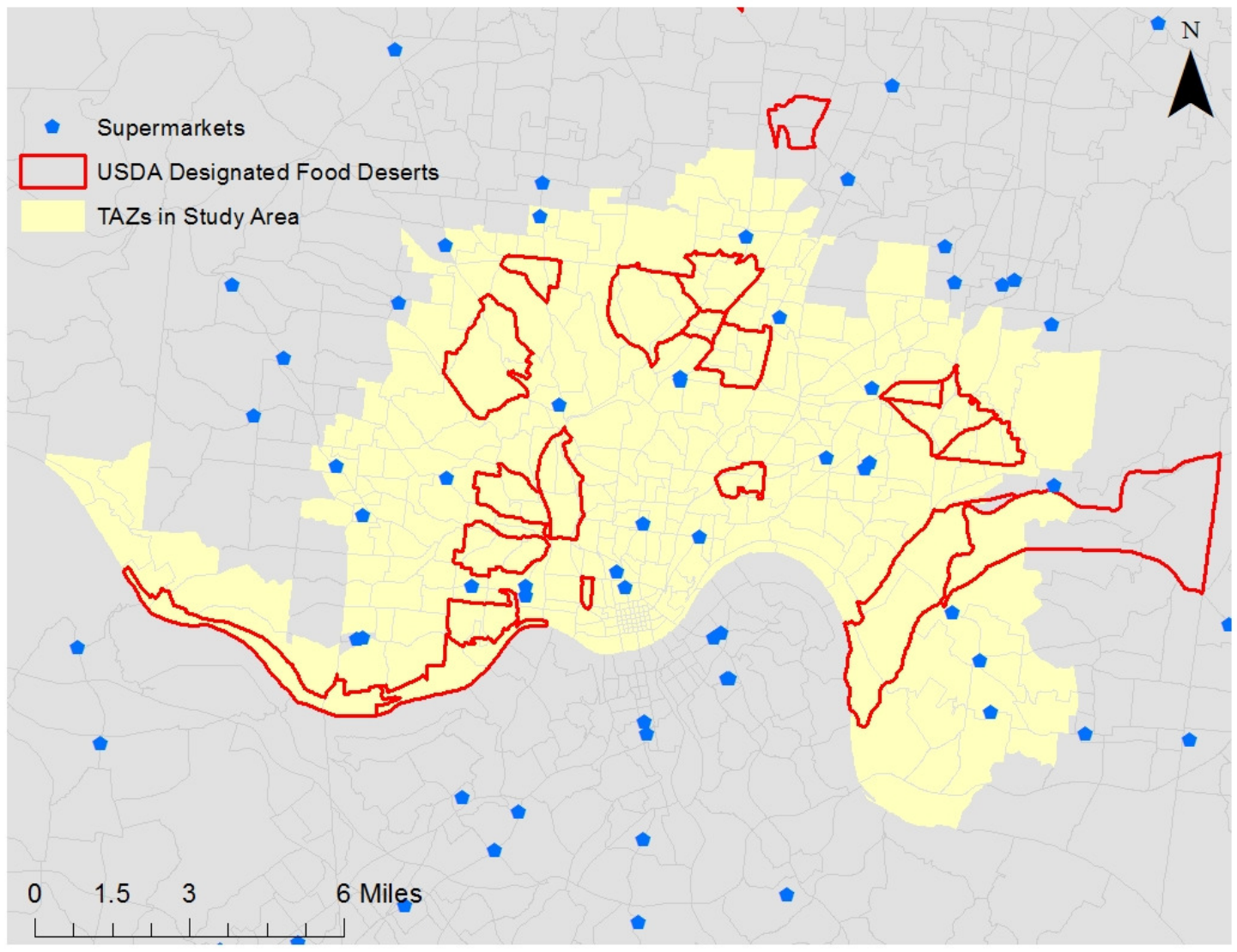

Figure 2. Map of study area. The focus of this study is on the residents who live in the yellow TAZs and commute to any of the MPO region TAZs.

have $B$ minutes available. While $S M I P_{i j k}$ provides an accessibility measure for a very specific combination of resident, work, and supermarket locations, it is desirable to more generally characterize the access of every residential TAZ, given their residents' varied commuting destinations.

This is achieved by calculating the following equation:

$S M I P_{i}=\sum_{j} P_{j}^{i} \frac{\sum_{k \in K} S M I P_{i j k}}{n}$ 
where $P_{j}^{j}$ denotes the proportion of commuters in TAZ $i$ who work in TAZ $j$ and $K$ denotes the set of $n$ supermarkets that yield the largest $S M I P_{i j}$ scores for commuters living in TAZ $i$ and commuting to $\mathrm{TAZ}_{j}$. The resulting $S M I P_{i}$ score thus represents the average time residents of TAZ $i$ have to shop at the $n$ most accessible supermarkets, given the residents' commuting patterns.

\subsection{Home-to-supermarket Interaction Potential}

In addition to the $S M I P_{i}$, a comparable score is developed where the interaction potential value represents the number of minutes for shopping a resident has given they only travel to and from a supermarket from their home TAZ. This home-to-supermarket interaction potential score $(H I P)$ corresponds to the home-based food desert metrics described in the introduction (Larsen and Gilliland, 2008; Lee and Lim, 2009; Raja et al., 2008). The HIP for a TAZ $i$ and supermarket $k$ is calculated as follows:

$H I P_{i k}=\max \left(0, B-\left(t_{i k}+t_{k i}\right)\right)$

where $B$ is the time budget allotted for grocery shopping and $t_{i k}$ and $t_{k i}$ are the travel times to and from the supermarket TAZ $k$ for residents living in TAZ $i$. To compute a more general score that corresponds to $\operatorname{SMIP}_{i}$, the $H I P_{i}$ score is calculated like so:

$H I P_{i}=\frac{\sum_{i=\kappa} H I P_{i k}}{n}$

which is simply the average time in minutes a resident in TAZ $i$ can spend at the set of $n$ supermarkets contained in $K$ that yield the largest $H I P_{i}$ scores for commuters living in TAZ $i$. 
A script is written in Python, which computes the $S M I P_{i}$ and $H I P_{i}$ scores considering the data of Cincinnati, with an assumed post-work free time budget $B$ of 120 minutes. With the results calculated, the data are mapped using ArcGIS 10 and graphed using Python tools.

\section{Results}

The following section explores the aggregate and local-level characteristics of the interaction potential scores described above.

\subsection{Spatial Distribution of Interaction Potential}

The $S M I P_{i}$ scores are mapped in Figure 3. TAZs near downtown Cincinnati have higher $\operatorname{SMIP}_{i}$ scores of 111 to 115 minutes, with scores of 106 to 110 emerging east of downtown. The lowest scores are present in TAZs in the periphery, most likely due to longer commute

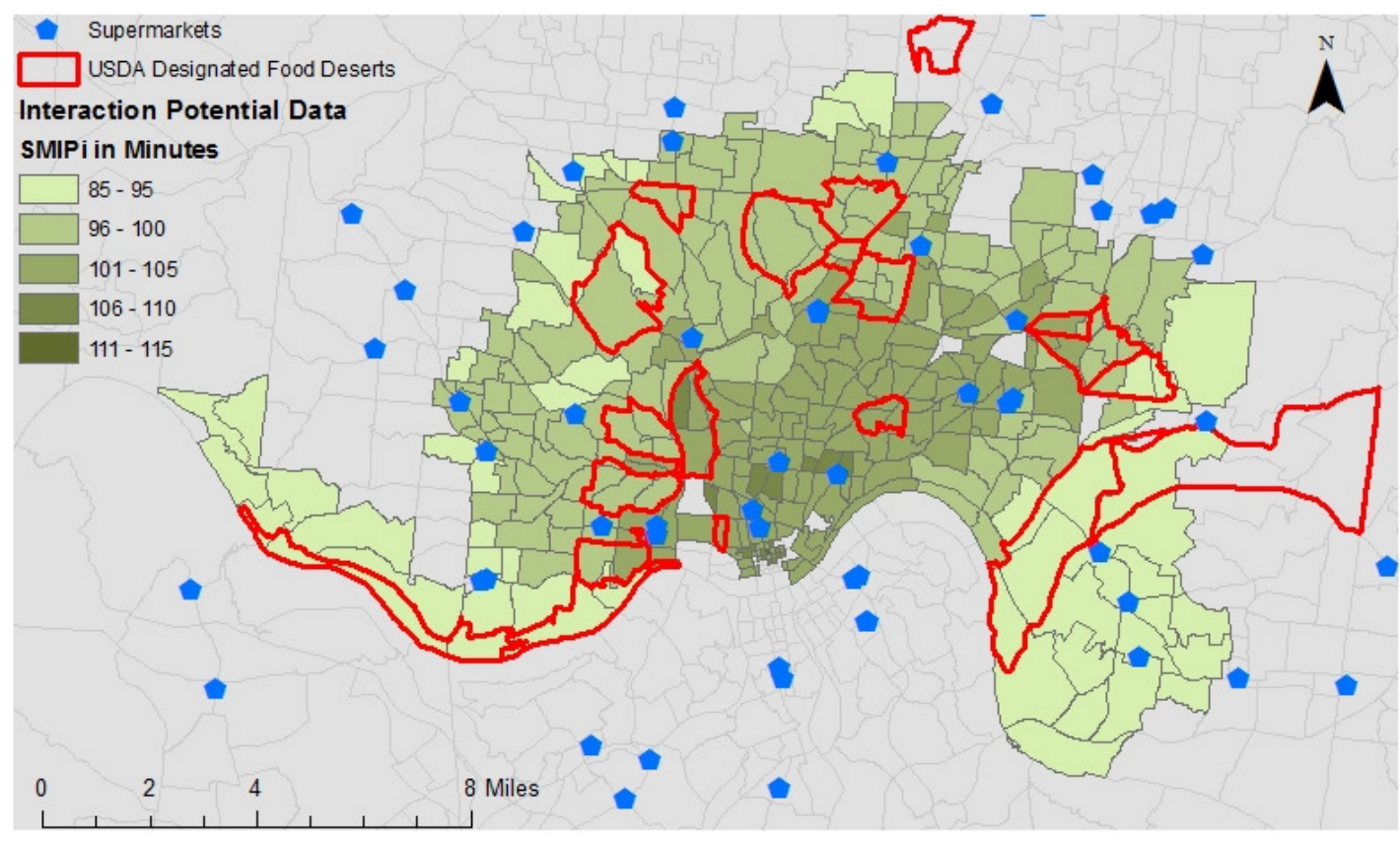

Figure 3. $S M I P_{i}$ scores for TAZs in Cincinnati, Ohio. 
times to TAZs with higher concentrations of jobs. Interestingly, TAZs that intersect with census tracts designated as food deserts by the USDA have a mean $S M I P_{i}$ score of 98.12 minutes $(\mathrm{n}=44, \mathrm{SD}=3.34)$ while the mean of the $S M I P_{i}$ scores for all Cincinnati TAZs is 89.58 minutes $(n=359, S D=29.25)$. While the greater interaction potential scores for food desert TAZs is unexpected, there are several reasons why this may be the case. First, given the high number of low-scoring TAZs, the mean for the entire study area may not be a good measure of central tendency. Additionally and as previously discussed, the USDA uses a threshold measure based on income and supermarket distance to determine a tract's food desert status, while the SMIP does not consider socio-economic disadvantages. Therefore, many of the low scoring TAZs in the region may have high socioeconomic status. Finally, the USDA uses a cut-off of one mile in their definition of food deserts, ignoring opportunities that exist just beyond this threshold.

Figure 4 contains a map of the comparable home-based $H I P_{i}$ scores. Downtown Cincinnati again has the highest scores of around 111 to 115 minutes, with lower scores radiating out from the city center. However, with this measure, some of the peripheral TAZs 


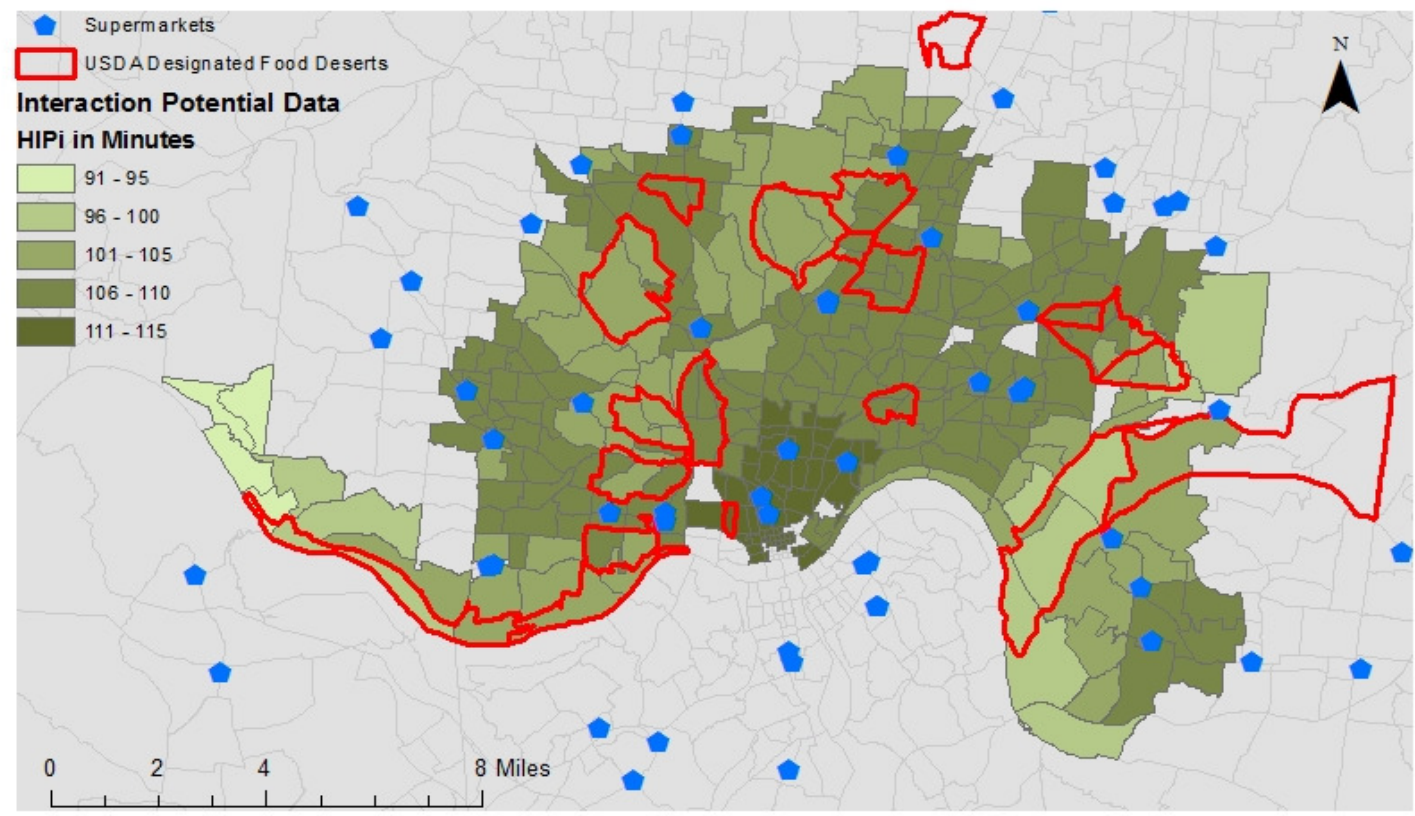

Figure 4. $H I P_{i}$ scores for TAZs in Cincinnati, Ohio.

with the lowest $S M I P_{i}$ scores have higher scores thanks to the removal of the commuting constraint. In other words, residents living in the city's outer TAZs, many of which are nearby a supermarket, must make longer commutes from the city's central business district, thus reducing their $S M I P_{i}$ scores. However, if residents in these TAZs only travel to and from their home TAZ, there is more time available for grocery shopping at nearby supermarkets.

To gain greater insight into the effect of automobile commuting on access to supermarkets, it is useful to create a ratio of $S M I P_{i}$ and $H I P_{i}$ scores. The resulting index of accessibility takes on values greater than one when the $S M I P_{i}$ score is larger than the $H I P_{i}$ score for a TAZ, values equal to one when $S M I P_{i}$ and $H I P_{i}$ are equal, and values of less than one when $S M I P_{i}$ is less than $H I P_{i}$.

Figure 5 shows the resulting index. The majority of TAZs have a ratio of less than one, implying that most residents have more time for grocery shopping trips to supermarkets if they do not chain the trip to their commute. However, two TAZs have a $S M I P_{i} / H I P_{i}$ ratio 
greater than one. For these two TAZs, residents' commutes increase their level of access to supermarkets, and interestingly, one of the two TAZs is located within a USDA food desert.

\subsection{Relationship Between the Average Top Five SMIP ${ }_{i j k}$ Scores and Commuting Costs}

While the spatial analysis presented in the previous section provides insights into how access varies across the city of Cincinnati, it fails to capture some of the nuances occurring within the residential TAZs. In particular, it is difficult to geographically map how the accessibility of workers living in the same TAZ but working in different TAZs varies for all zones in the study area. However, it is possible to plot the average of the five highest $S M I P_{i j k}$ scores for commuters living in TAZ $i$ and working in TAZ $j$ against the commuting costs incurred by traveling from $i$ to $j$. Figure 6 shows this relationship for all $i, j$ pairs, with a thirddegree least squares polynomial line fit to the data from every TAZ. For more on this method see Fan (1996). A degree of three is used because it is hypothesized there will be an initial increase in accessibility, followed by a decrease, and then a gradual approach to some $S M I P_{i j k}$ minimum, for a total of four constraints. The initial increase is posited because, for many TAZs, a short commute may place commuters closer to a supermarket while not depleting a large part of their time budget. The expected subsequent decrease corresponds to diminishing returns presented by a longer commute. A commuter that works further from their home will need to spend a considerable amount of their time budget only on getting to and from work, leaving less time to chain a trip to a supermarket. However, when the average of the top five $S M I P_{i j k}$ scores for all $i, j$ pairs are plotted, an almost linear negative relationship between the scores and commuting costs appears. The lack of the hypothesized non-linear trend implies that an initial increase in access due to commuting is not generalizable at the city-level. 


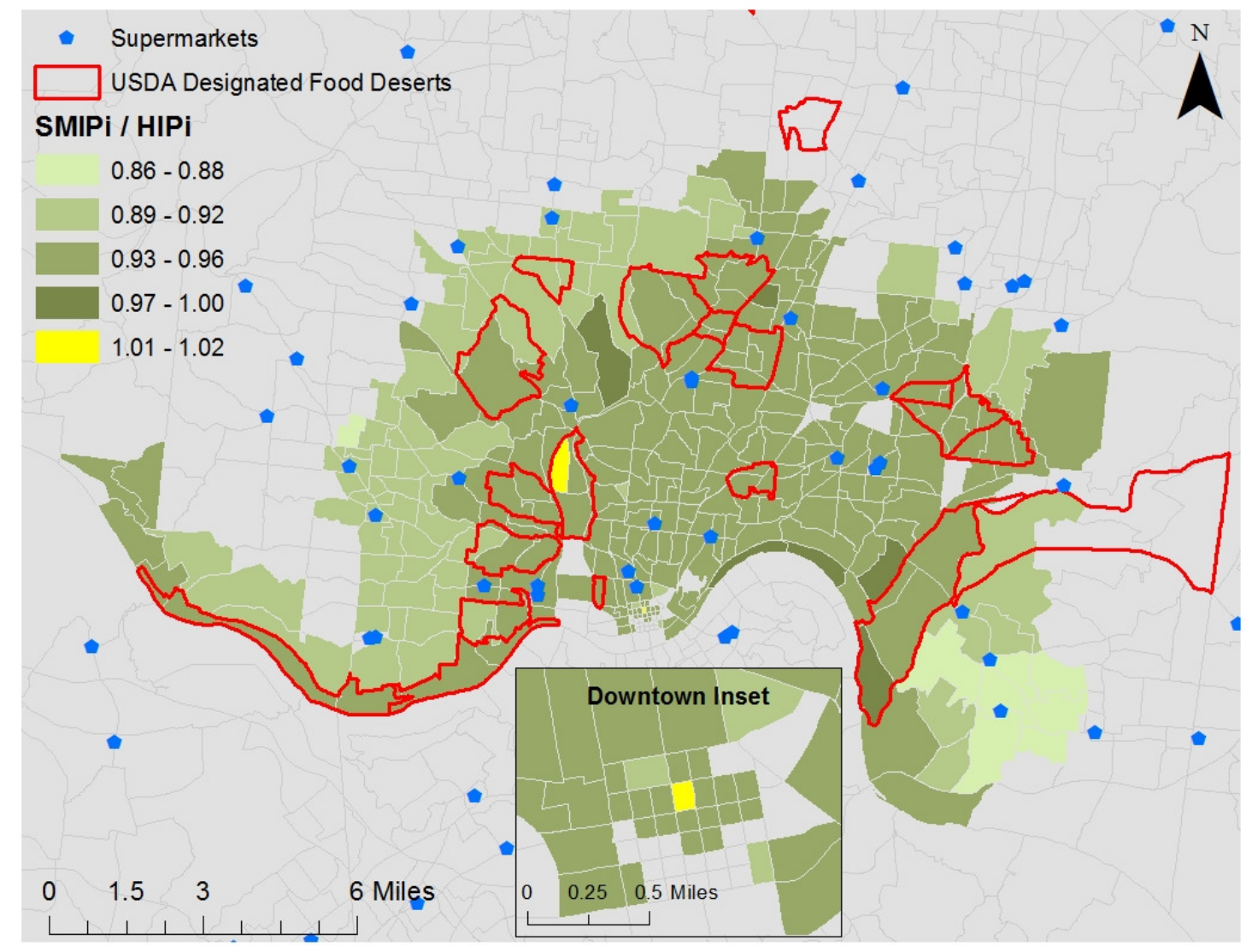

Figure 5. Ratio of the $S M I P_{i}$ and $H I P_{i}$ scores in Cincinnati, Ohio.

A closer inspection of the data is presented in Figures $\mathbf{7}$ and $\mathbf{8}$, with a third-degree least-squares polynomial fit line calculated for the $i, j$ pairs for each TAZ, where the number of commuters was greater than ten in order to generate useful fit lines. Additionally, in Figure 7 the data are categorized by median incomes of the home TAZ and Figure 8 presents TAZs that intersect with a USDA food desert. These classifications are included in order to investigate whether commuting costs have different effects on access to supermarkets for commuters of different income groups, or for those in food deserts. Here, visualization of the point data is excluded for clarity.

It becomes apparent in Figures $\mathbf{7}$ and $\mathbf{8}$ that the hypothesized non-linear trend does exist for some TAZs, with the average of the top five $S M I P_{i j k}$ scores increasing until a 
commuting cost of around 10 to 20 minutes. Additionally, there do not appear to be differences in the non-linear trend or the magnitude of scores across income categories, or food desert TAZs. These graphs show that commuters in some TAZs that chain a grocery shopping trip at a supermarket onto their drive home from work initially increase their access by having a longer commute time, but there are no clear differences in this non-linear trend across income groups.

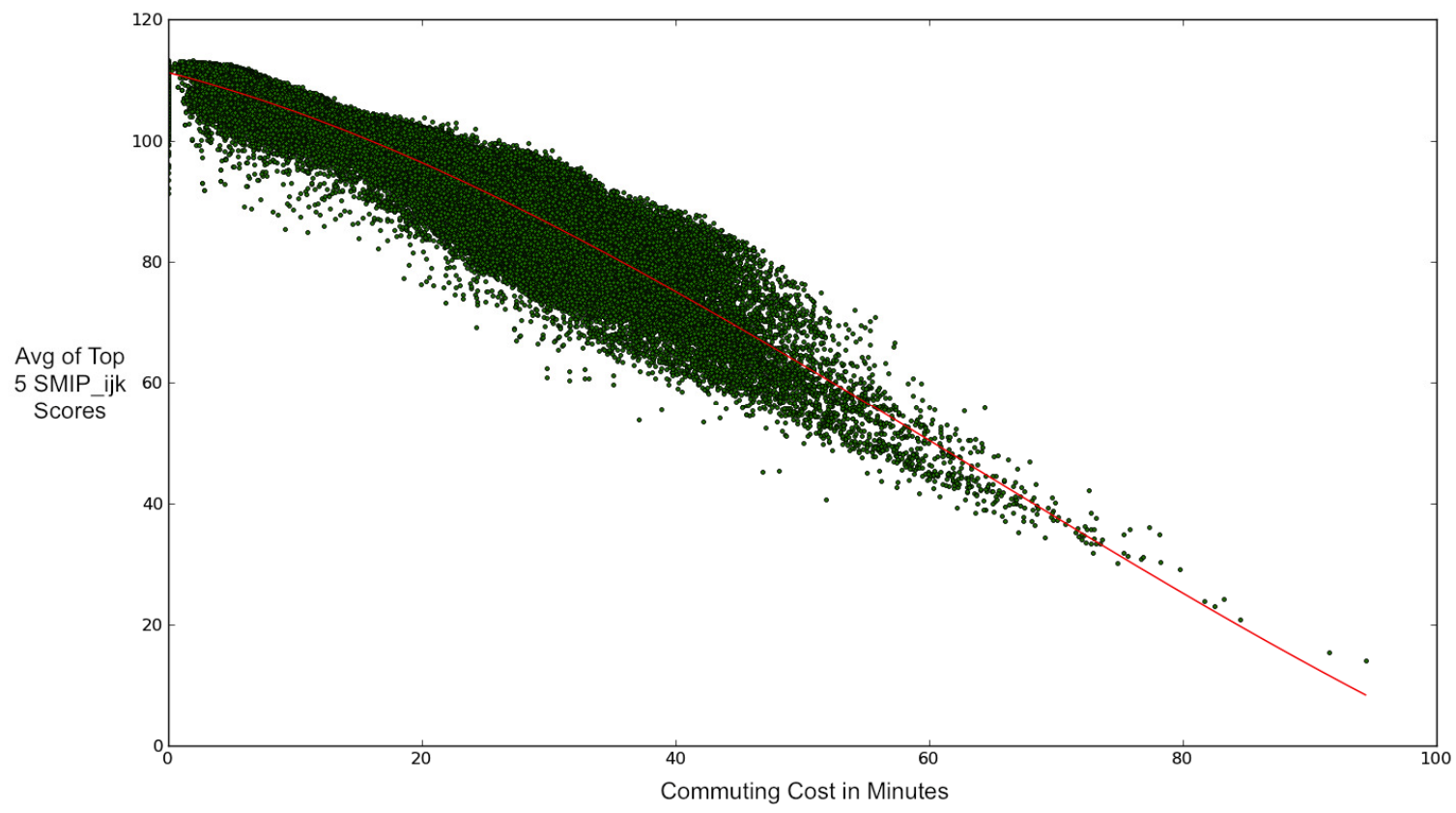

Figure 6. The relationship between commuting costs and the average of the top five $S M I P_{i j k}$ scores for all $i, j$ pairs. 

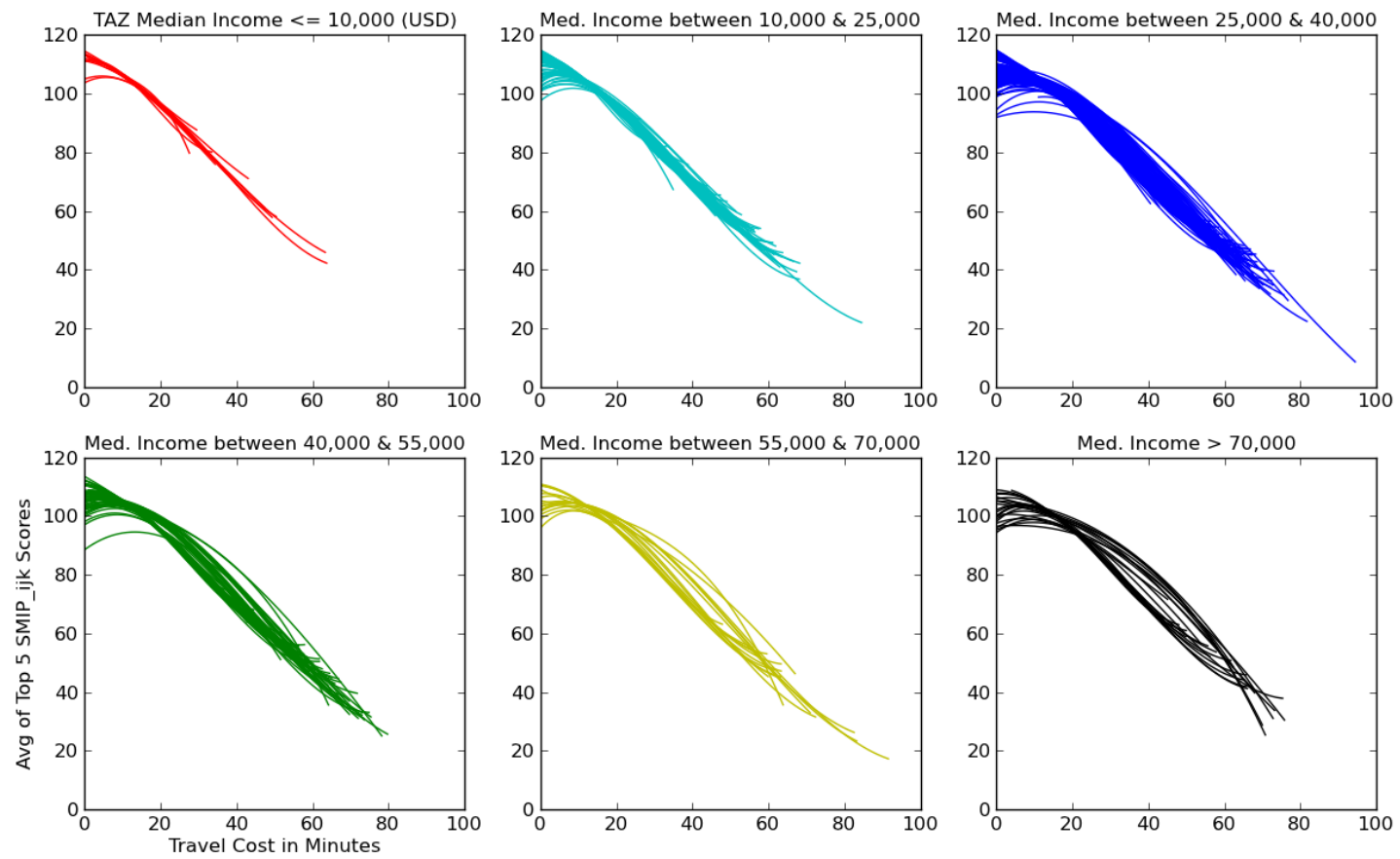

Figure 7. Average of the five largest $S M I P_{i j k}$ scores versus commuting cost for each $i, j$ pair.

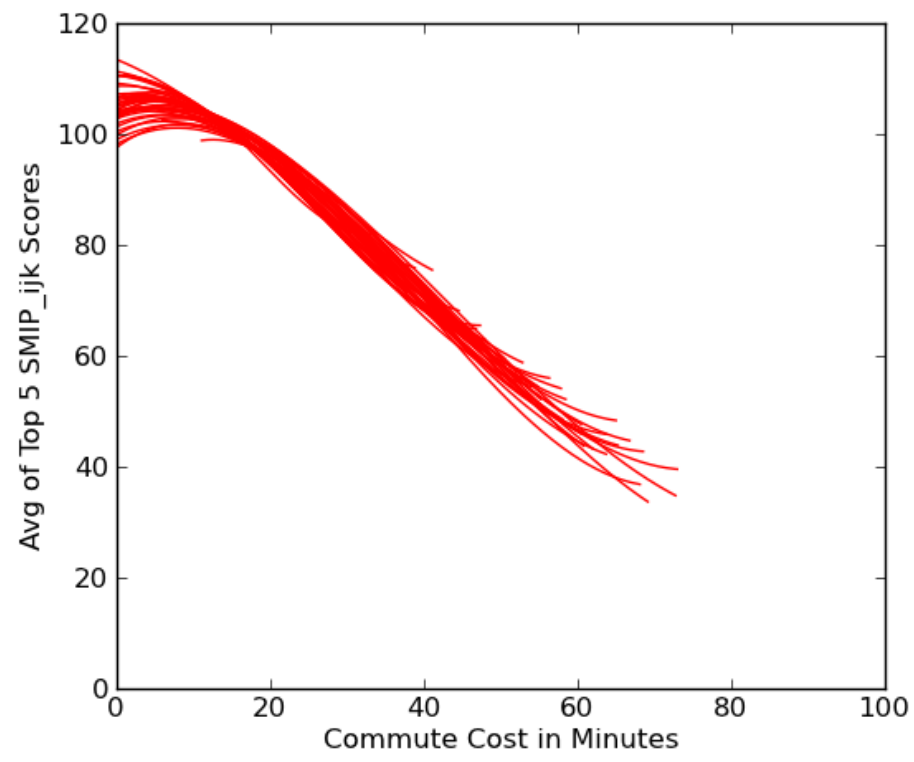

Figure 8. Average of the five largest $S M I P_{i j k}$ scores for TAZs that intersect with USDA food deserts. 
Finally, Figure 9 shows the probability distributions of commuting costs for the average of the five largest $S M I P_{i j k}$ scores for all TAZs, as well as TAZs that intersect with a USDA designated food desert. The average of the five largest $S M I P_{i j k}$ scores corresponds to the maximum point of the fit lines presented in Figures $\mathbf{7}$ and $\mathbf{8}$. More simply, what this graph shows is the probability that the best $S M I P_{i j k}$ scores from the general population of

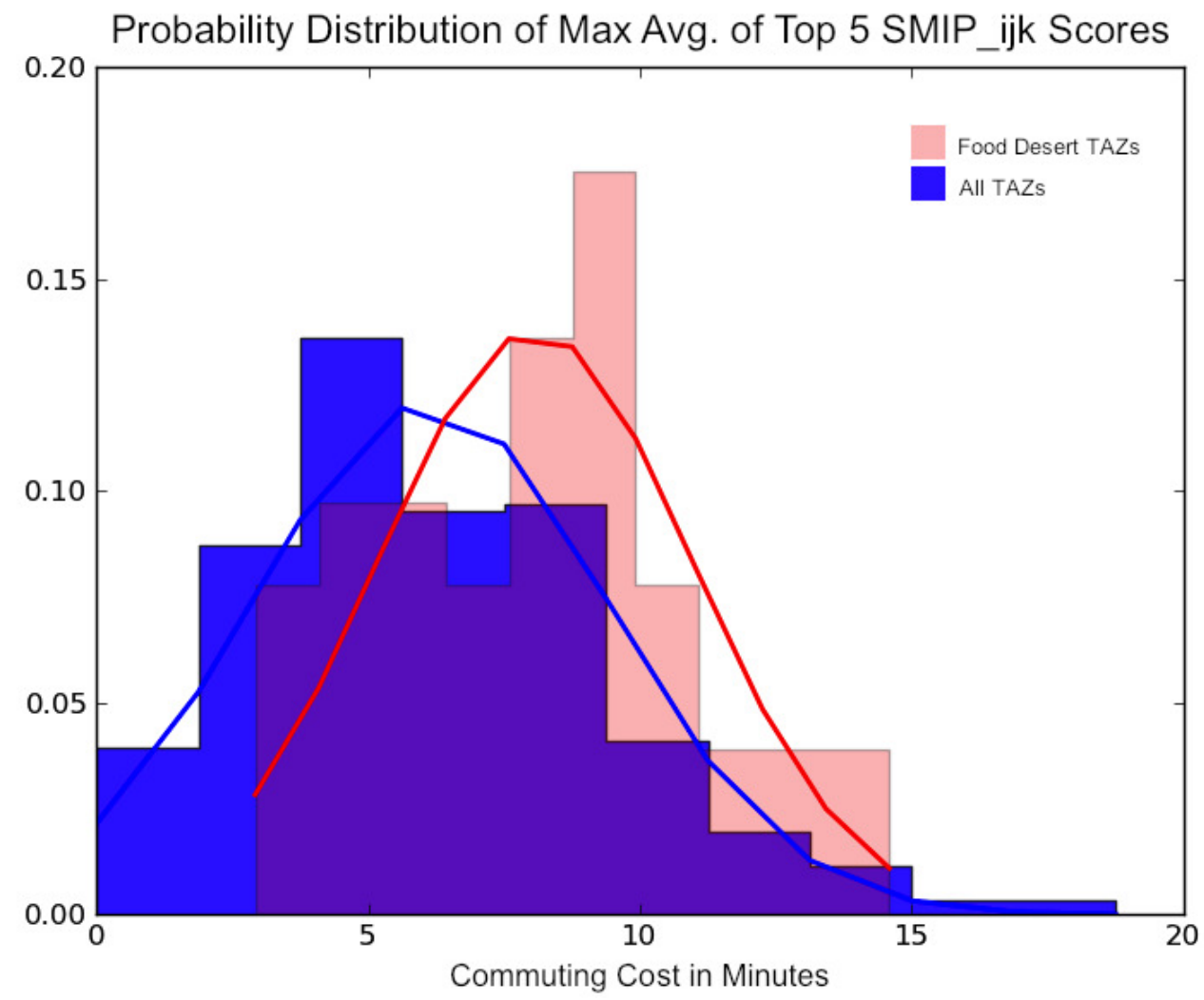

Figure 9. Probability distribution of commuting costs for the maximum average of the top five $S M I P_{i j k}$ scores for each TAZ with commuters. The lines are a fitted normal distribution, for reference.

TAZs and food desert TAZs are associated with various commuting costs from home to work. Doing this reveals that the distribution of maximum scores for TAZs associated with food deserts is shifted to the right of the distribution for all TAZs, which is not immediately 
apparent in the previous figures. This implies that given the current structure of commuting in Cincinnati, food desert TAZs initially benefit more from longer commutes to work than do TAZs in general. In other words, there is an observed initial increase in accessibility for commuters who travel further from home and reside in food desert TAZs.

\section{Discussion}

Numerous researchers have explored the geography of access to healthy food vendors, and most concede that spatial proximity is only one factor in a person's decision to shop at these locations (McKinnon et al., 2009). Cultural preference, food costs, and availability are equally important components. However, much of this research has had difficulty relating the concept of spatial access to health outcomes (An and Sturm, 2012). One reason for this could be that previous work has consistently employed methods to capture accessibility that greatly simplifies the reality experienced by residents.

The analysis presented in this paper provides an alternative measure that captures some of the population dynamics of an urban region by considering commuter flows, and reports a score representative of the average time residents have to grocery shop. Incorporating commuter flows accounts for a resident's expanded spatial reach achieved through daily movements, which would not be captured using an accessibility measure that only considers the home location. At the same time, the costs of commuting are incorporated into the SMIP metric thus balancing the costs (time-use) and benefits (spatial access) of the daily commute. This is in line with the core concepts of the time-geographic approach to modeling accessibility. Additionally, a related home-based measure with an equivalent output, the HIP, is derived to provide a benchmark for the novel commuting-based SMIP score. 
In the case of Cincinnati, Ohio, there are substantial differences in the spatial distribution of relatively good access that appear when using the SMIP (Figure 3), and the HIP (Figure 4). While, the HIP measure shows that trips to the supermarket originating from home generally result in more time available for grocery shopping, there are two instances where TAZs have more access thanks to daily commuting patterns. This analysis shows that population movements over time complicate the practice of measuring healthy food accessibility solely from residents' home locations.

When examining the average of the top five $S M I P_{i j k}$ scores for each TAZ, many zones benefit from a short 5-15 minute commute, which results in an increased amount of time available for grocery shopping. However, this trait is not present for all TAZs and is not found to be generalizable across the study area. Additionally, there appear to be no patterns across different income groups.

Finally, the probability of a TAZ's maximum average of top five $S M I P_{i j k}$ scores is compared with commuting costs. Here, the comparison shows that TAZs intersecting with USDA food desert tracts are more likely to have their maximum average of the top five $S M I P_{i j k}$ scores associated with a longer commute than are all TAZs in the study area. This implies that for these food desert TAZs, a longer car commute has the potential to initially increase access to supermarkets, and thus improve opportunities for purchasing healthy foods.

\section{Conclusions}

The focus of previous research on using home residences as the base for measuring access to food stores limits the understanding of where people have opportunities to purchase healthy foods. While there is evidence that urban residents tend to shop in their own neighborhoods or nearby town centers in Ireland (Furey et al., 2001), less is known about 
food shopping trends in the U.S. Even if shopping from home is preferred for reasons like familiarity and convenience, it is possible that daily population flows create an avenue for addressing spatial inaccessibility for some populations. This study presents a novel metric for measuring spatial access to healthy foods and shows that populations exist where commuting patterns increase the amount of access a person has to supermarkets.

It is important to note that this research aims to further the discussion on healthy food accessibility measures by serving as a proof of concept. As such, there are several limitations. With lack of better data to guide our selection, the post-work time-budget has been introduced exogenously as 120 minutes. Future data collection is needed to justify this budget, or else the study could be repeated for different time-budgets in a sensitivity analysis. The division of labor within a household may result in a commuting worker not being the primary grocery shopper, and therefore reduce the impact of any additional opportunities gained through daily movements. Additionally, some commuters may not pursue these additional opportunities for a number of reasons. Perhaps they have a regularly scheduled weekend grocery trip, or are unwilling to spend additional time running this errand after a full day of work. Despite this, the time-geographic accessibility measure presented in this paper demonstrates that a more complete representation of healthy food access reveals opportunities that are not captured in static metrics. By using this method to identify new potential opportunities, intervention strategies could be proposed that incentivize these postwork trips.

It is hypothesized that moving toward healthy food accessibility measures that more realistically represent people's exposure to the environment throughout the day will eliminate some of the ambiguity found in previous studies. For example, some research has found low levels of home-based spatial access does not always lead to less nutritious household diets (An and Sturm, 2012; Macintyre, 2007). By fully understanding the spatial movements of 
grocery shoppers, it will be possible to disentangle the role of geography from other socioeconomic factors. This is imperative if appropriate and successful policy measures are to be developed.

\section{References}

Alwitt, L.F., Donley, T.D., 1997. Retail stores in poor urban neighborhoods. Journal of consumer affairs 31, 139-164.

An, R., Sturm, R., 2012. School and Residential Neighborhood Food Environment and Diet Among California Youth. American Journal of Preventive Medicine 42, 129-135.

Bertrand, L., Thérien, F., Cloutier, M., 2008. Measuring and mapping disparities in access to fresh fruits and vegetables in Montreal. Canadian Journal of Public Health 99.

Cervero, R., 1996. Mixed land-uses and commuting: evidence from the American Housing Survey. Transportation Research Part A: Policy and Practice 30, 361-377.

Cox, A.M., McKevitt, C., Rudd, A.G., Wolfe, C.D.A., 2006. Socioeconomic status and stroke. The Lancet Neurology 5, 181-188.

Cummins, S., Macintyre, S., 2002. A systematic study of an Urban Foodscape: The Price and Availability of Food in Greater Glasgow. Urban Studies 39, 2115-2130.

ERS USDA, 2012. Food Desert Locator. http://www.ers.usda.gov/data/fooddesert/.

Everson, S.A., Maty, S.C., Lynch, J.W., Kaplan, G.A., 2002. Epidemiologic evidence for the relation between socioeconomic status and depression, obesity, and diabetes. Journal of psychosomatic research 53, 891-895.

Fan, J., 1996. Local Polynomial Modelling and Its Applications. Monographs on Statistics and Applied Probability. Chapman \& Hall/CRC.

Farber, S., Neutens, T., Miller, H., Li, X., In Press. The Social Interaction Potential of Metropolitan Regions: a Time-Geographic Measurement Approach Using Joint Accessibility. Annals of the Association of American Geographers.

Furey, S., Strugnell, C., McIlveen, M.H., 2001. An investigation of the potential existence of 'food deserts"in rural and urban areas of Northern Ireland. Agriculture and Human Values 18, 447-457.

Hendrickson, D., Smith, C., Eikenberry, N., 2006. Fruit and vegetable access in four lowincome food deserts communities in Minnesota. Agriculture and Human Values 23, 371383.

Horner, M.W., 2004. Spatial Dimensions of Urban Commuting: A Review of Major Issues and Their Implications for Future Geographic Research. The Professional Geographer 56, $160-173$.

Horner, M.W., O'Kelly, M.E., 2007. Is non-work travel excessive? Journal of Transport Geography 15, 411-416. 
Hung, H.C., Joshipura, K.J., Jiang, R., Hu, F.B., Hunter, D., Smith-Warner, S.A., Colditz, G.A., Rosner, B., Spiegelman, D., Willett, W.C., 2004. Fruit and vegetable intake and risk of major chronic disease. Journal of the National Cancer Institute 96, 1577-1584.

Joshipura, K., Hu, F., Manson, J., Stampfer, M., Rimm, E., Speizer, F., Colditz, G., Ascherio, A., Rosner, B., Spiegelman, D., 2001. The effect of fruit and vegetable intake on risk for coronary heart disease. Annals of Internal Medicine 134, 1106.

Larsen, K., Gilliland, J., 2008. Mapping the evolution of 'food deserts' in a Canadian city: Supermarket accessibility in London, Ontario, 1961 - 2005. International Journal of Health Geographics 7, 16.

Lee, G., Lim, H., 2009. A Spatial Statistical Approach to Identifying Areas with Poor Access to Grocery Foods in the City of Buffalo, New York. Urban Studies 46, 1299.

Lopez, R.P., 2007. Neighborhood Risk Factors for Obesity. Obesity 15, 2111-2119.

Macintyre, S., 2007. Deprivation amplification revisited; or, is it always true that poorer places have poorer access to resources for healthy diets and physical activity. International Journal of Behavioral Nutrition and Physical Activity 4, 1-7.

McKenzie, B., Rapino, M., 2011. Commuting in the United States: 2009 - American Community Survey Reports. United States Census Bureau.

McKinnon, R.A., Reedy, J., Morrissette, M.A., Lytle, L.A., Yaroch, A.L., 2009. Measures of the Food Environment: A Compilation of the Literature, 1990-2007. American Journal of Preventive Medicine 36, 124-133.

Metcalf, S., Widener, M.J., 2012. Growing Buffalo's capacity for local food: A systems framework for sustainable agriculture. Journal of Applied Geography.

Neutens, T., Delafontaine, M., Scott, D.M., Maeyer, P.D., In Press. An analysis of day-to-day variations in individual space-time accessibility. Journal of Transport Geography.

Orbis, 2012. Company Information. Bureau Van Dijk. http://www.bvdinfo.com/products/company-information/international/orbis.

Páez, A., Mercado, R.G., Farber, S., Morency, C., Roorda, M., 2010. Relative accessibility deprivation indicators for urban settings: definitions and application to food deserts in Montreal. Urban Studies 47, 1415-1438.

Popkowski Leszczyc, P.T.L., Sinha, A., Sahgal, A., 2004. The effect of multi-purpose shopping on pricing and location strategy for grocery stores. Journal of Retailing 80, 8599.

Powell, L., Slater, S., Mirtcheva, D., Bao, Y., Chaloupka, F., 2007. Food store availability and neighborhood characteristics in the United States. Preventive Medicine 44, 189-195.

Powell, L., Han, E., Zenk, S.N., Khan, T., Quinn, C.M., Gibbs, K.P., Pugach, O., Barker, D.C., Resnick, E.A., Myllyluoma, J., Chaloupka, F.J., 2011. Field validation of secondary commercial data sources on the retail food outlet environment in the U.S. Health and Place 17, 1122-1131.

Raja, S., Ma, C., Yadav, P., 2008. Beyond food deserts: measuring and mapping racial disparities in neighborhood food environments. Journal of Planning Education and Research 27, 469.

Salze, P., Banos, A., Oppert, J., Charreire, H., Casey, R., Simon, C., Chaix, B., Badariotti, D., Weber, C., 2011. Estimating spatial accessibility to facilities on the regional scale: an 
extended commuting-based interaction potential model. International Journal of Health Geographics 10, 2.

Schafft, K.A., Jensen, E.B., Hinrichs, C.C., 2009. Food Deserts and Overweight

Schoolchildren: Evidence from Pennsylvania*. Rural Sociology 74, 153-177.

Shaw, H., 2006. Food deserts: Towards the development of a classification. Geografiska Annaler: Series B, Human Geography 88, 231-247.

US Department of Health and Human Services, 2010. Healthy People 2020.

US Census, 2012. 2009-2011 American Community Survey.

Walker, R.E., Keane, C.R., Burke, J.G., 2010. Disparities and access to healthy food in the United States: A review of food deserts literature. Health \& Place 16, 876-884.

Widener, M.J., Metcalf, S.S., Bar-Yam, Y., 2011. Dynamic Urban Food Environments: A Temporal Analysis of Access to Healthy Foods. American Journal of Preventive Medicine 41, 439-441.

Widener, M.J., Metcalf, S.S., Bar-Yam, Y., 2012. Developing a Mobile Produce Distribution System for Low-income Urban Residents in Food Deserts. Journal of Urban Health In Press.

Wrigley, N., 2002. 'Food Deserts' in British Cities: Policy Context and Research Priorities. Urban Studies 39, 2029-2040. 